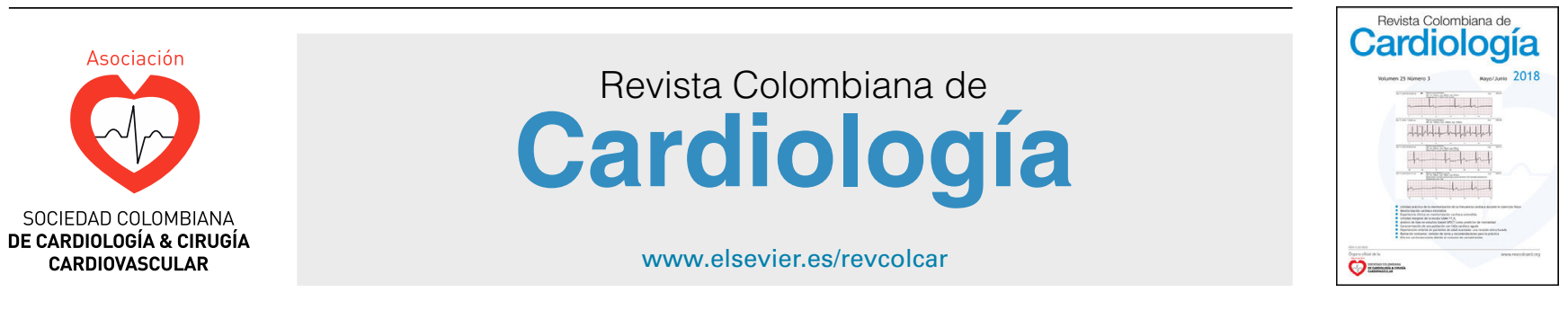

ARTÍCULO ESPECIAL

\title{
Riesgo cardiovascular relacionado con la radiación ionizante
}

\author{
Julián M. Aristizábal
}

CES University - Unit of Cardiología Medellin, Colombia

Recibido el 16 de septiembre de 2019; aceptado el 17 de noviembre de 2019

\section{PALABRAS CLAVE \\ Efectos de la radiación; Enfermedades cardiovasculares; Protección a radiación; Exposición ocupacional}

\section{KEYWORDS}

Effects of radiation; Cardiovascular diseases; Radiation protection; Occupational exposure

\begin{abstract}
Resumen Nuevas investigaciones han planteado un vínculo previamente no valorado lo suficiente, radiación ionizante y enfermedad cardiovascular, puesto que el interés se ha centrado en la radiación y el cáncer. El desarrollo en terapias oncológicas ha favorecido un aumento en la supervivencia de muchos pacientes, pero, por otro lado, ha influenciado el perfil de riesgo cardiovascular de los mismos: la exposición a la radioterapia torácica está asociada con enfermedad coronaria e incremento del riesgo de eventos cardiovasculares. Así mismo, existe evidencia incipiente que demuestra la relación entre exposición crónica a dosis moderadas o bajas de radiación y enfermedad cardiovascular.

(c) 2019 Sociedad Colombiana de Cardiología y Cirugía Cardiovascular. Publicado por Elsevier España, S.L.U. Este es un artículo Open Access bajo la licencia CC BY-NC-ND (http:// creativecommons.org/licenses/by-nc-nd/4.0/).
\end{abstract}

\section{Cardiovascular risk related to ionizing radiation}

Abstract Recent studies have established a link between ionising radiation and cardiovascular disease. This has not been sufficiently assessed before, as interest has focused on radiation and cancer. The development of cancer treatments has led to an increase in survival of many patients, but on the other hand, they have had an influence on the cardiovascular risk profile of many of them. The exposure to chest radiotherapy is associated with coronary disease and an increase in the risk of cardiovascular events. Furthermore, there is growing evidence that demonstrates the association between long-term exposure to moderate or low doses of radiations and cardiovascular disease.

(c) 2019 Sociedad Colombiana de Cardiología y Cirugía Cardiovascular. Published by Elsevier España, S.L.U. This is an open access article under the CC BY-NC-ND license (http:// creativecommons.org/licenses/by-nc-nd/4.0/). 


\section{Introducción}

Tradicionalmente se han considerado los problemas ortopédicos, el cáncer o las cataratas como los principales riesgos ocupacionales en médicos que trabajan con fluoroscopia y radiación ionizante. Nuevas investigaciones plantean un vínculo previamente no valorado de manera suficiente: radiación ionizante y enfermedad cardiovascular. El desarrollo en terapias oncológicas ha aumentado la supervivencia de muchos pacientes, pero, por otra parte, ha influenciado el perfil de riesgo cardiovascular de los mismos: la exposición a la radioterapia torácica está asociada con enfermedad coronaria e incremento del riesgo de eventos cardiovasculares ${ }^{1}$. Así mismo, existe evidencia incipiente que demuestra la relación entre exposición crónica a dosis moderadas o bajas de radiación y enfermedad cardiovascular ${ }^{2}$.

\section{¿Es un problema real?}

El personal médico y paramédico de un laboratorio de cateterización cardíaca puede acumular durante su vida laboral una exposición a radiación ionizante en un rango entre 50 a $200 \mathrm{mSv}$, con un exceso atribuible en el riesgo de cáncer de 1 en $100^{3}$. Existe coincidencia en algunos de los mecanismos que explican los daños cardiovasculares provocados por las altas dosis de radiación (radioterapia torácica, exposición a centrales nucleares, sobrevivientes de bombas nucleares, entre otros) con aquellos atribuidos a la radiación ionizante en dosis moderadas o bajas. Esta relación justifica la inquietud acerca del nexo entre radiación ocupacional y enfermedad cardiovascular, así como la aparición de varios estudios que brindan información interesante: una revisión reciente que menciona múltiples estudios epidemiológicos reporta un exceso de riesgo en enfermedad coronaria y cerebrovascular con rangos de exposición entre 0,5 y $5 \mathrm{~Gy}^{4}$.

En relación a grupos de trabajadores de la salud con exposición a radiación ionizante también existe información novedosa; en una cohorte de trabajadores canadienses expuestos a diversas formas de radiación se encontró un riesgo de mortalidad cardiovascular aumentado, proporcional a la dosis de exposición. De igual forma, en un grupo de tecnólogos de imagen en Estados Unidos expuestos a procedimientos guiados por fluoroscopia se reportó un aumento del $34 \%$ en la incidencia de accidente cerebrovascular en comparación con quienes no trabajaron en estos procedimientos. Sin embargo, la posibilidad de factores de confusión debe tenerse en cuenta puesto que un estudio adicional que compara médicos psiquiatras con médicos que realizan algún tipo de fluoroscopia no encontró diferencias en la mortalidad cardiovascular ${ }^{5-7}$.

Específicamente en personal de laboratorio de cateterismo cardíaco, Andreassi et al. encontraron un aumento en el grosor íntima-media y un acortamiento de la longitud de los telómeros en los leucocitos, ambos marcadores de daño vascular precoz ${ }^{8}$. Aunque el perfil de riesgo de los evaluados difería parcialmente de los controles, la presencia de marcadores específicos de daño vascular alerta sobre el vínculo presupuestado. De manera complementaria, la presencia de biomarcadores que reflejan el daño al ADN en trabajadores expuestos a radiación ionizante corrobora los hallazgos previos y contribuye a la comprensión de los mecanismos explicativos ${ }^{9}$. Incluso, un estudio posterior de los mismos autores reporta una prevalencia mayor de hipertensión y dislipidemia en personal con exposición crónica a la radiación ionizante (principalmente cardiólogos intervencionistas y electrofisiólogos), conectando las bases fisiopatológicas y los hallazgos de laboratorio con expresiones clínicas y enfermedades cuya presencia puede estar aumentada por este factor ${ }^{10}$. Llamativamente no hubo mayor presencia de eventos adversos cardiovasculares. Por supuesto, atribuir directamente la dislipidemia por la exposición a la radiación aún resulta complejo y, más bien, consiste en un llamado de atención en posibles mecanismos que se sobreponen y modifican el perfil del riesgo del trabajador de salud expuesto a radiación. En este orden de ideas, la tamización temprana y el inicio de terapias que modifiquen el curso de la enfermedad puede ser deseable.

Aunque algunas de las versiones disponibles pueden sugerir incongruencias es preciso recordar la interacción compleja de factores que posibilitan el desarrollo de la enfermedad cardiovascular y cómo la modulación de los factores de riesgo afecta la expresión clínica de la misma. Ahora, la posible vinculación con el desarrollo de enfermedad aterosclerótica precoz asociada con disfunción endotelial, fenómenos inflamatorios, remodelación y aumento de la trombogenicidad constituye una base teórica razonable para explicar el riesgo cardiovascular incrementado y el desarrollo posterior de eventos clínicos, como los accidentes cerebrovasculares.

\section{¿Cuáles son los mecanismos?}

Los efectos de la radiación ionizante pueden categorizarse en tres: de bajas dosis $(<100 \mathrm{mSv})$, de dosis moderadas (100-1000 mSv) y de altas dosis (>1Sv) que usualmente se observan en exposición a radioterapia ${ }^{11}$. Aunque para el desarrollo del cáncer no existe una dosis mínima, para el desarrollo de enfermedad cardiovascular se requirieron exposiciones bajas a moderadas. A pesar de considerar el sistema cardiovascular un sistema altamente diferenciado, con bajas tasas mitóticas, hace varias décadas se conoce el efecto deletéreo de la exposición a la radiación, observado inicialmente con los pacientes expuestos a radioterapia. Dado el desarrollo de métodos diagnósticos y terapéuticos complejos que utilizan fluoroscopia en dosis elevadas, la inquietud se trasladó a los operadores y al personal paramédico expuesto: ¿tienen estas personas un riesgo cardiovascular mayor relacionado con la radiación ionizante? Los datos arrojados por varios estudios alrededor del mundo plantean una respuesta afirmativa y empiezan a catalogar este aumento del riesgo cardiovascular como un riesgo ocupacional ${ }^{10}$.

Los daños potenciales pueden tener diversas expresiones: enfermedad aterosclerótica acelerada, envejecimiento vascular prematuro, enfermedad valvular, afectación pericárdica, miocardiopatía y disfunción autonómica.

Los mecanismos relacionados son igualmente variados ${ }^{1}$ :

- Ruptura de las cadenas de ADN mediante daño directo o por radicales libres: la medición de marcadores de daño y reparación del ADN fue realizada recientemente en intervencionistas que hacían procedimientos de 
reparación endovascular de aorta. La presencia de marcadores de daño con la exposición a la radiación ionizante y la reducción de estos al utilizar medidas de radioprotección fueron hallazgos muy relevantes en la investigación ${ }^{12}$. Adicionalmente, las variaciones genéticas que favorecen una reparación temprana del ADN con pequeños daños pueden modificar la expresión final de la enfermedad.

- Afectación de los procesos de señalización intracelular: variación en niveles y expresión de citoquinas, moléculas de adhesión y leucocitos.

- Fibrosis: aumento de permeabilidad vascular, coagulación, degradación de la membrana endotelial, diferenciación temprana de fibroblastos y depósito de colágeno.

- Estrés oxidativo: regulación a la baja de sintetasa del óxido nítrico y desregulación mitocondrial.

- Alteraciones procoagulantes: expresión de factor de Von Willebrand, aumento en los niveles de factor tisular y regulación a la baja de la trombomodulina ${ }^{11}$.

- Cambios en la extensión de los telómeros: el acortamiento de los telómeros en los leucocitos puede llevar a un daño irreversible del material genético con posterior envejecimiento y apoptosis al inhibir la capacidad de reparación celular ${ }^{13}$. Varios estudios han relacionado el acortamiento de los telómeros con el desarrollo de enfermedad coronaria, infarto de miocardio y eventos cerebrovasculares ${ }^{14}$. De igual forma, algunos factores externos, entre ellos la radiación ionizante, han sido asociados como explicación potencial al nexo entre enfermedad aterosclerótica acelerada y radiación ${ }^{8}$.

\section{Medidas de protección}

El principio ALARA (del inglés, as low as reasonably achievable $)^{15}$ continúa siendo la base del manejo de riesgo con la exposición a la radiación ionizante, tanto para los operadores, como para los pacientes. Las alternativas para reducir la dosis de radiación recibida usualmente han logrado demostrar resultados favorables: una estrategia con el acrónimo ALARA demostró la reducción en la dosis recibida en operadores y pacientes pediátricos sometidos a ablación de arritmias $^{16}$. Recientemente un estudio de la Clínica Cleveland reportó una reducción del $70 \%$ en las dosis recibidas en los operadores (electrofisiólogos) durante un período de once años ${ }^{17}$, mientras que un estudio en un centro de referencia en Italia refleja nuevamente reducción en la dosis recibida pero además demuestra cómo la variación entre los operadores es marcada y debe tenerse en cuenta en los lineamientos de una política institucional con el fin de hacer homogéneos los resultados y los potenciales beneficios ${ }^{18}$. La educación del personal en formación es una alternativa valiosa: los ambientes de entrenamiento con simuladores pueden tener impacto favorable en la dosis recibida por el operador en su práctica cotidiana $^{19}$.

El desarrollo de técnicas complejas de intervención plantea un nuevo reto: las altas dosis de radiación en procedimientos como las intervenciones para reparo endovascular aórtico con la demostración de daño agudo en el ADN del operador $^{12}$ o las ablaciones complejas de fibrilación auricular o taquicardia ventricular constituyen una situación con potencial mayor de daño relacionado con la radiación ionizante, pero también la oportunidad para probar nuevas medidas de protección, nuevas tecnologías de imagen que reduzcan la fluoroscopia y herramientas simplificadas para reducir los tiempos del procedimiento, que al mismo tiempo beneficien la morbilidad y mortalidad de pacientes más enfermos.

Aunque la reducción de la dosis recibida es un elemento medible y constituye un objetivo trazable, el impacto de tales medidas en el desarrollo de enfermedad cardiovascular resulta más complejo de evaluar: el peso de los factores de confusión y los riesgos competitivos en una enfermedad multiforme, condicionada por variables e intervenciones diversas, que requieren un tiempo prolongado de exposición para manifestarse clínicamente, hace realmente difícil discriminar el impacto específico de las medidas de radioprotección.

La medición de biomarcadores que reflejen el daño relacionado con la radiación ionizante es una estrategia potencialmente útil dado que se involucra específicamente en la fisiopatogenia de los procesos que llevan al daño celular y tisular: los marcadores de daño y reparación del $A D N$, el acortamiento telomérico, los marcadores citogenéticos de exposición de susceptibilidad ${ }^{20}$, entre otros, podrían aumentar la precisión en la detección temprana de daños y facilitarían la instauración de medidas para remediarlo. Esto, por supuesto, implica tecnología novedosa no disponible de manera rutinaria, pero puede constituir una de las formas más adecuadas de seguimiento para un proceso dinámico y complejo.

Por otro lado, el papel de los medicamentos para prevenir específicamente los efectos de la radiación en el lecho vascular está por definirse; el uso de terapias con impacto en el sistema renina-angiotensina-aldosterona, con acción en la función endotelial y disminución de la trombogenicidad, y efectos potenciales antiinflamatorios, resulta atractivo desde una perspectiva teórica, pero requiere estudios a largo plazo. Entre las limitaciones fundamentales para demostrar la utilidad de tales terapias se encuentran la alta prevalencia de las enfermedades en la población general, el impacto de otros múltiples factores en la progresión de las mismas, el uso de marcadores subrogados para definir una respuesta a la terapia, así como factores de confusión más difíciles de sedimentar y excluir de los análisis de eficacia.

\section{Conclusiones}

Existe evidencia inicial que avala un proceso fisiopatológico previamente no considerado como relevante, que se expresa clínicamente como un mayor riesgo y enfermedad cardiovacular en el personal de salud expuesto a radiación ionizante. Aunque los datos son tempranos, son plausibles y se nutren de nuevos marcadores muy específicos. Estos hallazgos deben ser evaluados rigurosamente y tomados en cuenta con el fin de instaurar y reforzar las medidas de radioprotección necesarias, para evitar que se siga considerando la aparición de enfermedades relacionadas con la radiación en el personal médico o paramédico, como una anécdota infortunada. 


\section{Creative Commons}

Creative Commons Reconocimiento-No Comercial-Sin Obra Derivada (cc BY-NC-ND).

\section{Conflicto de intereses}

Ninguno.

\section{Bibliografía}

1. Spetz J, Moslehi J, Sarosiek K. Radiation-induced cardiovascular toxicity: mechanisms, prevention, and treatment. Curr Treat Options Cardiovasc Med. 2018;20:31.

2. Little MP. Radiation and circulatory disease. Mutat Res. 2016;770 Pt B:299-318.

3. Vano E, Gonzalez L, Fernandez JM, Alfonso F, Macaya C. Occupational radiation doses in interventional cardiology: a 15-year follow-up. Br J Radiol. 2006;79:383-8.

4. Kreuzer M, Auvinen A, Cardis E, Hall J, Jourdain JR, Laurier D, et al. Low-dose ionising radiation and cardiovascular diseasesStrategies for molecular epidemiological studies in Europe. Mutat Res Rev Mutat Res. 2015;764:90-100.

5. Zielinski JM, Ashmore PJ, Band PR, Jiang H, Shilnikova NS, Tait VK, et al. Low dose ionizing radiation exposure and cardiovascular disease mortality: cohort study based on Canadian national dose registry of radiation workers. Int J Occup Med Environ Health. 2009;22:27-33.

6. Linet MS, Kitahara CM, Ntowe E, Kleinerman RA, Gilbert ES, Naito N, et al. Mortality in U.S. Physicians Likely to Perform Fluoroscopy-guided Interventional Procedures Compared with Psychiatrists, 1979 to 2008. Radiology. 2017;284:482-94.

7. Rajaraman P, Doody MM, Yu CL, Preston DL, Miller JS, Sigurdson $\mathrm{AJ}$, et al. Incidence and mortality risks for circulatory diseases in US radiologic technologists who worked with fluoroscopically guided interventional procedures, 1994-2008. Occup Environ Med. 2016;73:21-7.

8. Andreassi MG, Piccaluga E, Gargani L, Sabatino L, Borghini A, Faita F, et al. Subclinical carotid atherosclerosis and early vascular aging from long-term low-dose ionizing radiation exposure: a genetic, telomere, and vascular ultrasound study in cardiac catheterization laboratory staff. JACC Cardiovasc Interv. 2015;8:616-27.

9. Borghini A, Mercuri A, Turchi S, Chiesa MR, Piccaluga E, Andreassi MG. Increased circulating cell-free DNA levels and mtDNA fragments in interventional cardiologists occupationally exposed to low levels of ionizing radiation. Environ Mol Mutagen. 2015;56:293-300.

10. Andreassi MG, Piccaluga E, Guagliumi G, Del Greco M, Gaita F, Picano E. Occupational Health Risks in Cardiac Catheterization Laboratory Workers. Circ Cardiovasc Interv. 2016;9: e003273.

11. Borghini A, Gianicolo EA, Picano E, Andreassi MG. Ionizing radiation and atherosclerosis: current knowledge and future challenges. Atherosclerosis. 2013;230:40-7.

12. El-Sayed T, Patel AS, Cho JS, Kelly JA, Ludwinski FE, Saha P, et al. Radiation-Induced DNA Damage in Operators Performing Endovascular Aortic Repair. Circulation. 2017;136:2406-16.

13. Fyhrquist F, Saijonmaa O, Strandberg T. The roles of senescence and telomere shortening in cardiovascular disease. Nat Rev Cardiol. 2013;10:274-83.

14. D'Mello MJ, Ross SA, Briel M, Anand SS, Gerstein H, Pare G. Association between shortened leukocyte telomere length and cardiometabolic outcomes: systematic review and metaanalysis. Circ Cardiovasc Genet. 2015;8:82-90.

15. Hirshfeld JW, Ferrari VA, Bengel FM, Bergersen L, Chambers CE, Einstein AJ, et al. 2018 ACC/HRS/NASCI/SCAI/SCCT Expert Consensus Document on Optimal Use of Ionizing Radiation in Cardiovascular Imaging-Best Practices for Safety and Effectiveness, Part 2: Radiological Equipment Operation, Dose-Sparing Methodologies. Patient and Medical Personnel Protection: A Report of the American College of Cardiology Task Force on Expert Consensus Decision Pathways. J Am Coll Cardiol. 2018;71:2829-55.

16. Gellis LA, Ceresnak SR, Gates GJ, Nappo L, Pass RH. Reducing patient radiation dosage during pediatric SVT ablations using an "ALARA" radiation reduction protocol in the modern fluoroscopic era. Pacing Clin Electrophysiol. 2013;36:688-94.

17. Wunderle KA, Chung MK, Rayadurgam S, Miller MA, Obuchowski NA, Lindsay BD. Occupational and patient radiation doses in a modern cardiac electrophysiology laboratory. J Interv Card Electrophysiol. 2018, http://dx.doi.org/10.1007/ s10840-018-0462-8.

18. Casella M, Dello Russo A, Russo E, Catto V, Pizzamiglio F, Zucchetti M, et al. X-Ray Exposure in Cardiac Electrophysiology: A Retrospective Analysis in 8150 Patients Over 7 Years of Activity in a Modern. Large-Volume Laboratory. J Am Heart Assoc. 2018;7.

19. Katz A, Shtub A, Solomonica A, Poliakov A, Roguin A. Simulator training to minimize ionizing radiation exposure in the catheterization laboratory. Int J Cardiovasc Imaging. 2017;33:303-10.

20. Hall J, Jeggo PA, West C, Gomolka M, Quintens R, Badie C, et al. lonizing radiation biomarkers in epidemiological studies - An update. Mutat Res. 2017;771:59-84. 